\title{
Development of a liquid scintillator based active fission target for FIPPS
}

\author{
F. Kandzia ${ }^{1, \mathrm{a}}$, G. Belier ${ }^{2}$, C. Michelagnoli ${ }^{1, b}$, J. Aupiais ${ }^{2}$, M. Barani ${ }^{3}$, J. Dudouet ${ }^{4}$, Ch. E. Düllmann ${ }^{5,6,7}$, \\ Ł. W. Iskra ${ }^{8}$, M. Jentschel ${ }^{1}$, Y. H. Kim ${ }^{1}$, U. Köster ${ }^{1}$, A. Turturica ${ }^{9}$ \\ ${ }^{1}$ Institut Laue Langevin, 71 Avenue des Martyrs, 38042 Grenoble, France \\ 2 CEA, DAM, DIF, 91297 Arpajon, France \\ ${ }^{3}$ Dipartimento di Fisica, Universita degli Studi di Milano, Via Festa del Perdono 7, 20122 Milan, Italy \\ ${ }^{4}$ Université Lyon 1, CNRS/IN2P3, IPN-Lyon, 69622 Villeurbanne, France \\ 5 Johannes Gutenberg-Universität Mainz, 55099 Mainz, Germany \\ ${ }^{6}$ GSI Helmholtzzentrum für Schwerionenforschung GmbH, 64291 Darmstadt, Germany \\ ${ }^{7}$ Helmholtz Institut Mainz, 55099 Mainz, Germany \\ ${ }^{8}$ INFN sezione di Milano, Via Celoria 16, 20133 Milan, Italy \\ ${ }^{9}$ Horia Hulubei National Institute of Physics and Nuclear Engineering-IFIN HH, 077125 Bucharest, Romania
}

Received: 14 April 2020 / Accepted: 12 July 2020 / Published online: 18 August 2020

(C) The Author(s) 2020

Communicated by Navin Alahari

\begin{abstract}
An active fission target has been developed for the FIPPS instrument at ILL, enabling for the first time an efficient suppression of $\beta$-delayed $\gamma$ rays in high-resolution and high-efficiency $\gamma$-ray spectroscopy of fission fragments at a neutron beam. The target is based on a liquid scintillator in which the actinide is dissolved, resulting in a $4 \pi$ fragment detection. Measurements have been performed with ${ }^{233,235} \mathrm{U}$, with a fission tagging efficiency of $97.8(25) \%$. The high efficiency, together with the good time resolution of the scintillator target, provide high-selectivity data for $\gamma$-ray spectroscopy studies of fission fragments.
\end{abstract}

\section{Introduction}

In the last decades, $\gamma$-ray spectroscopy with germanium detector arrays has been established as a leading method for the determination of the structure of nuclei [1]. Various mechanisms are available to produce nuclei in excited states, one being nuclear fission. The spectroscopy of prompt $\gamma$ rays from fission fragments allows to study neutron-rich isotopes in the mass range $80<A<160$, with average spin states around 6-7 $\hbar$ [2] and values up to $20 \hbar$ [3]. In the past, extensive studies have been performed with spontaneous fission sources e.g. with the EUROGAM/EUROBALL [4] and GAMMASPHERE [3,5] arrays as well as with neutroninduced fission, e.g. in the EXILL campaign [6] and heavy-

\footnotetext{
a e-mail: kandzia@ill.fr (corresponding author)

b e-mail: michelagnolic@ill.fr (corresponding author)
}

ion induced fission $[7,8]$. In this kind of investigations, the identification of a given fission fragment relies on multiple $\gamma$-ray coincidence techniques. For this selection method to be efficient, high resolution, granularity and peak-over-total ratio are required from the $\gamma$-ray spectrometer. One limiting aspect of such studies, especially when investigating isotopes with small fission yields, is the $\gamma$-ray background originating from $\beta$ decays of radioactive fission products, particularly compromising the quality of the coincidence energy spectra. In the past fission tagging devices have been developed for measurements of fission and neutron capture cross sections of actinides [9-11], fission $\gamma$-ray calorimetry [12,13] and measurements of fission neutron distributions $[14,15]$. The direct detection of one or both fission fragments has been achieved e.g. with fission ionization chambers $[9,14]$, parallel plate avalanche counters [10,12], Micromegas [11], scintillating gases [15] or thin scintillating films [13]. Although the fission tagging detectors have been versatile for fission studies, they are not suitable for $\gamma$-ray spectroscopy due to the long flight-path of the fission fragments, causing Doppler shifts of the emitted $\gamma$ rays. In this work, the implementation of a fission detector for high-resolution $\gamma$-ray spectroscopy following thermal neutron induced fission is reported. An efficient suppression of $\beta$-delayed $\gamma$ rays from fission products has been possible for the first time by using a liquid scintillator based active fission target which was developed for the FIPPS spectrometer, located at the Institut Laue-Langevin (ILL) in Grenoble, France. The Fission Product Prompt $\gamma$-ray Spectrometer (FIPPS) [16] is an instrument for the study of 
the structure of nuclei produced in thermal neutron induced reactions. The neutrons are produced by the institute's high flux reactor and delivered as a strongly collimated beam to the target position, with a beam diameter of about $15 \mathrm{~mm}$ and a capture equivalent flux of up to $10^{8} \mathrm{n} / \mathrm{cm}^{2} / \mathrm{s}$. The main spectrometer consists of eight germanium clover detectors which form a closed octagon around the target, perpendicular to the neutron beam. Additional clover detectors can be installed (up to 16 clovers in total) to achieve a higher solid angle coverage. In order to provide a fission tagging detector for FIPPS, which can be deployed in the neutron beam, an active target was developed based on an organic liquid scintillator. The actinide of interest is directly dissolved in the scintillator, allowing for a $4 \pi$ fragment detection with high efficiency. This paper presents the design of the active target setup as well as the performance during the first fission campaigns, using ${ }^{233} \mathrm{U}$ and ${ }^{235} \mathrm{U}$ targets.

\section{Design of the active fission target}

The following constraints had to be considered for the design of the active fission target for FIPPS: (1) minimization of beam-induced $\gamma$-ray background, (2) minimization of $\gamma$-ray absorption between the target and the germanium detectors, (3) efficient scintillation light collection in a vacuum chamber around a neutron beam, (4) compliance with radioprotection requirements. While (1) mainly influenced the choice of materials, (2), (3) and (4) determined the geometry of the setup. The developed implementation is schematically depicted in Fig. 1 and is described in detail below. The active fission target setup is composed of three parts: (a) a target cell with the actinide-loaded scintillator, (b) a light guiding system to propagate the scintillation light to a photomultiplier tube (PMT), (c) a dedicated vacuum chamber.

(a) The actinide-loaded liquid scintillator has been developed at the Commissariat à l'Energie Atomique (CEA) Bruyères-le-Châtel (France) [18]. The high efficiency and good particle identification capabilities of this scintillator have already been demonstrated with a target for precision studies of spontaneous fission branching ratios [19]. For the present application, in order to minimize the background due to neutron capture on hydrogen, a deuterated version of the scintillator has been prepared, exploiting the smaller neutron capture cross section of deuterium ( $0.5 \mathrm{mb}$ compared to $332.6 \mathrm{mb}$ for ${ }^{1} \mathrm{H}$ [20]). The base solvent of the scintillator is deuterated toluene with additions of PBBO as primary fluor and deuterated naphthalene for an improved pulse shape discrimination (PSD) capability (the detailed composition and full names of chemicals are listed in Table 1). The PSD is based on the presence of a fast and a slow scintillation light component. The intensity of the latter depends on the ionizing power of the incident particle and thus allows to dis-

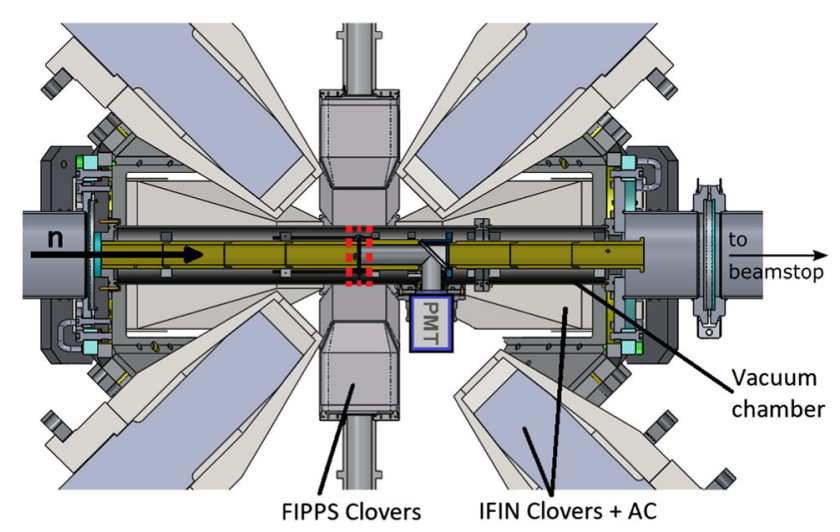

Fig. 1 Section view of the active target setup at the FIPPS instrument. The direction of the collimated, thermal neutron beam (left-to-right) is indicated by an arrow. Eight germanium clover detectors are arranged in the plane perpendicular to the neutron beam at the target position. Eight additional clover detectors with their anti-Compton (AC) shields (loan from IFIN-HH [17]) are mounted in horizontal and vertical 45deg positions with respect to this plane. The active target cell is mounted at target position (red dashed lines). It is optically connected to the PMT by a light guiding system, both are also shown. ${ }^{6} \mathrm{Li}$-loaded cylinders (represented in yellow) mounted around the neutron beam all along the vacuum chamber are used to absorb scattered neutrons, thus minimizing the $\gamma$-ray background

tinguish between weakly and strongly ionizing particles, i.e. electrons, alpha particles and fission fragments. The actinide loading of the scintillator is achieved by liquid-liquid extraction from a primary aqueous solution [21], performed at the CEA. The primary solution for the ${ }^{235} \mathrm{U}$ target has been prepared at the CEA, and that for the ${ }^{233} \mathrm{U}$ target at the Institute of Nuclear Chemistry at Johannes Gutenberg University Mainz. For the latter, $1 \mathrm{mg}$ of ${ }^{233} \mathrm{U}$ was dissolved in $8 \mathrm{M}$ hydrochloric acid and fed onto an anion exchange column (DOWEX AG 1X8) to first remove the daughters of its decay chain. The uranium was eluted with $0.5 \mathrm{M}$ hydrochloric acid, converted into the nitrate system, and then shipped to CEA. The extracting molecule, HDEHP, has been added into the scintillator mix. While at FIPPS only uranium targets have been used so far, a loading with other actinides is possible and may be considered for future studies. The target cell containing the scintillator is shown in Fig. 2. The materials and layer thicknesses (see Fig. 2a) have been chosen in order to comply with the requirements (1) and (2), listed above.

The central part of the cell consists of a sapphire window, exhibiting good mechanical stability and light transmission, a PTFE (Polytetrafluoroethylene - $\left.\left(\mathrm{C}_{2} \mathrm{~F}_{4}\right)_{n}\right)$ washer defining the thickness of the scintillator volume and a PTFE backing. A cyanoacrylate glue is used to assemble these three components, after a pre-treatment of the PTFE pieces with a commercially available primer solution. The PTFE backside is lined with an aluminized mylar foil in order to increase the reflectivity and maximize the scintillation light output through the sapphire window. A second sapphire disc 
Table 1 Composition of the liquid scintillator used for the active fission target

\begin{tabular}{lll}
\hline Molecule & Purpose & Concentration \\
\hline Toluene (deuterated) & Base solvent & \\
Naphthalene (deuterated) & PSD enhancer & $200 \mathrm{mg} / \mathrm{ml}$ \\
PBBO (2-(4-Biphenylyl)-6-phenyl-1,3-benzoxazole) & Fluor & $5 \mathrm{mg} / \mathrm{ml}$ \\
HDEHP (Bis(2-ethylhexyl) phosphoric acid) & Extracting molecule & $2 \mathrm{mg} / \mathrm{ml}$ \\
\hline
\end{tabular}

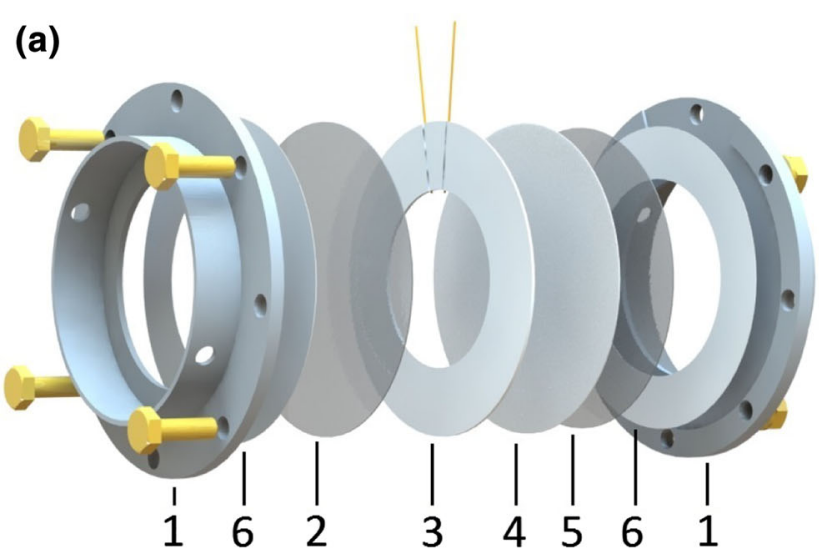

Fig. 2 a Layout of the target cell: (1) aluminum frame, (2) front sapphire window (thickness $0.2 \mathrm{~mm})$, (3) central PTFE washer $(0.5 \mathrm{~mm})$ with capillaries (inner diameter $0.3 \mathrm{~mm})$, (4) PTFE backing $(0.2 \mathrm{~mm})$, lined with an aluminized mylar foil, (5) supporting sapphire disc $(0.2$

mechanically supports the PTFE backing, to guarantee the flatness of the volume. This assembly is enclosed in between two aluminum clamps for mounting and mechanical stability (a picture of the ensemble is shown in Fig. 2b). Additional PTFE washers mechanically protect the sapphire windows against local pressure points from the aluminum frame. The outer diameter of the scintillator volume $(26 \mathrm{~mm})$ and the inner diameter of the aluminum frame $(30.5 \mathrm{~mm})$ are chosen sufficiently larger than the neutron beam diameter (about $15 \mathrm{~mm}$ ) in order to allow for uncertainties in the cell positioning without risking that structural materials intersect with the neutron beam. Two flexible capillary tubes are fed through the central PTFE washer into the inner cell volume to allow for filling and venting of the cell after its assembly. After filling, the tightness of the inner volume of the cell is achieved by clamping the capillaries. Before installation at FIPPS, the tightness of the cell was verified in order to avoid a potential contamination of the instrument. To this end a leakage test was performed with the sealed cell placed in vacuum, exploiting the high volatility of the scintillator.

(b) A light guiding system is required in order to read out the scintillation light with a conventional PMT, while minimizing the interference with the neutron beam and the amount of absorbing materials between the active target and the germanium detectors. The schematic representation of

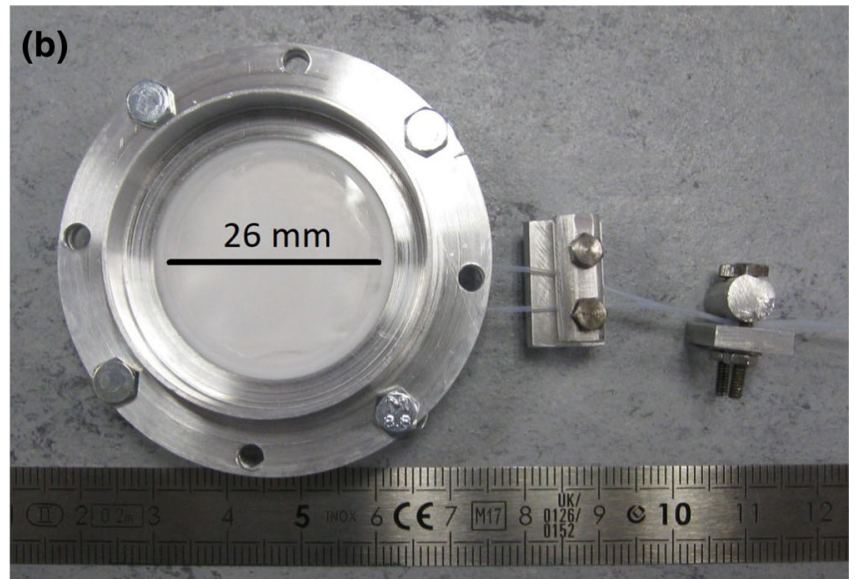

$\mathrm{mm})$, (6) PTFE washers (0.1 mm). See text for more details. b Target cell without scintillator. After filling the cell with the scintillator, the two capillaries are clamped (twice, for redundancy) in order to guarantee the tightness of the cell

this system is shown in Fig. 3. The scintillation light exiting the target cell through the front sapphire window is first guided in the direction of the neutron beam by a thin $(\approx$ $40 \mu \mathrm{m}$ ), specular reflective tube of $28 \mathrm{~mm}$ diameter. A thin mirror foil intersects the neutron beam at an angle of $45^{\circ}$, about $10 \mathrm{~cm}$ downstream from the target position. The light is thus reflected towards a viewport in the vacuum chamber, guided by a second reflective tube perpendicular to the first. A PMT is directly mounted onto the viewport. The reflective tubes are produced by rolling up DF2000MA foils from 3M [22]. DF2000MA is a metal-free, specular reflective polymer film with $>99 \%$ reflectivity for visible light over a large range of incident angles, at only $38 \mu \mathrm{m}$ thickness [22,23]. The mirror consists of an aluminized mylar foil of only few micrometers thickness, stretched over an elliptical frame.

(c) The vacuum chamber housing the target cell is made of a carbon fiber tube, aligned concentrically to the neutron beam and to the detector array by fixation to the HPGe clovers' support structure (see Fig. 1). The tube has a wall thickness of only $2 \mathrm{~mm}$ to minimize $\gamma$-ray absorption. Along its axis, concentric to the neutron beam, a set of ${ }^{6} \mathrm{Li}$-loaded plastic cylinders is installed in order to absorb scattered neutrons (Fig. 1). The target cell is mounted in between two of these cylinders, see Fig. 3. 


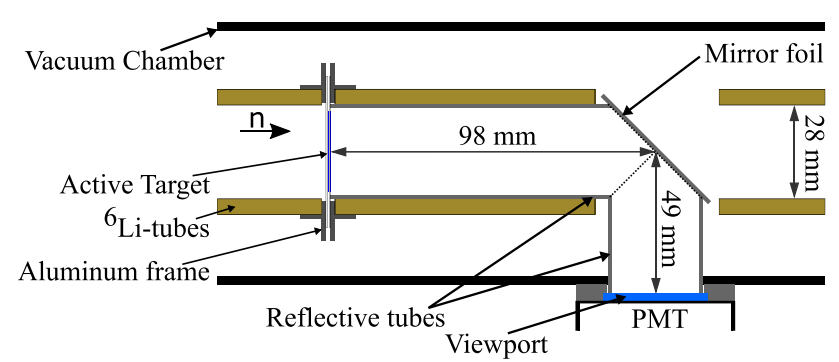

Fig. 3 Schematic view of the light readout system for the active target. The scintillation light exits the target cell through the downstream sapphire window, is guided by a highly reflective tube along the neutron beam to a mirror foil and finally is reflected towards a PMT

\section{Experiments at FIPPS}

Active targets following the above-described design were used for experiments at FIPPS with ${ }^{233,235} \mathrm{U}$ loaded scintillators. In these experiments the thermal-neutron induced fission is exploited to populate neutron-rich nuclei in the mass region $80<A<160$ and investigate a variety of nuclear structure phenomena. The first experimental campaign was performed with ${ }^{235} \mathrm{U}$. The loaded scintillator was prepared at the CEA and delivered to ILL with nominally $650 \mu \mathrm{g}$ of ${ }^{235} \mathrm{U}$ diluted in $370 \mu \mathrm{l}$ of scintillator. Uncertainties in the efficiency of the liquid-liquid extraction process and the occurrence of small residues during the cell filling procedure contribute to an indetermination in the target mass. In addition, the presence of air bubbles, unavoidably also sealed within the cell, compromises the exact prevision of the amount of actinide actually encountered by the beam. However, for the study of the structure of fission products, this information is not required. The analysis of the active target's performance during this first campaign is described in detail in the following section. In order to overcome the main limiting factor of this first prototype - the pile-up between fission and background signals, as will be shown below - a second version of the active target setup was developed. In order to achieve a maximum fission rate with a minimal signal pile-up, the active volume and the signal readout of the active target have to be sub-divided. To this end, the target assembly has been modified by replacing the supporting sapphire disc (Fig. 2a, piece 5) with an additional target cell, identical to the first one (Fig. 2a, pieces 2-4) and within the same aluminum frame, but oriented in opposite direction. A second light guiding system and PMT were installed in upstream direction from the target position to have independent signal readouts for the two cells, allowing to double the overall in-beam target mass without increasing the pile-up rate. This double cell setup was used in two more experimental campaigns of ther$\mathrm{mal}$ neutron induced fission of uranium isotopes, ${ }^{233} \mathrm{U}$ and

${ }^{235} \mathrm{U}$, where the latter was intended as a complement to the previous campaign and demonstrated the proof of principle of the setup, using the same mass of uranium. The data collected with the ${ }^{233} \mathrm{U}$ target unfortunately suffered from low statistics due to an error in the determination of the extracted mass. In the single-cell configuration with the ${ }^{235} \mathrm{U}$ target [24] a fission rate up to $12 \mathrm{kHz}$ was achieved with a total of 32 days beam on target, resulting in about $2 \times 10^{10}$ fission tagged $\gamma-\gamma$-coincidences. In the double-cell configuration [25] with the ${ }^{233} \mathrm{U}\left({ }^{235} \mathrm{U}\right)$ target a maximum fission rate of $4 \mathrm{kHz}(15 \mathrm{kHz})$ was achieved, with data being collected over 35 days (4 days).

\section{In-beam performance of the uranium-loaded active target}

For the time correlations among the scintillator signal and the germanium detectors to be available, the signals from the PMT have been recorded with the ILL standard electronics, namely with a Caen V1751 digitizer with 10-bit resolution and $1 \mathrm{Gs} / \mathrm{s}$ sampling rate. A charge integration over user-defined long- and short-time intervals is obtained as an output. The germanium clover detectors of the FIPPS array are read independently with Caen V1725 boards, designed for pulse-height analysis, with 14-bit resolution and $500 \mathrm{Ms} / \mathrm{s}$ sampling rate. The data recorded from the different boards are synchronized via a common clock. The data recording in list mode allows for an offline event reconstruction with versatile event-building options. The standard event definition is based on the coincidence of the PMT signal with at least one HPGe signal within a time window of $500 \mathrm{~ns}$. The event-time is associated with the timestamp of the PMT signal. The energy of the scintillation signal is determined by charge integration over a user-defined time interval. The total charge $\left(Q_{\text {tot }}\right)$ is extracted from an integration window of $60 \mathrm{~ns}(130 \mathrm{~ns}$ for the double cell setups), opened $10 \mathrm{~ns}$ before the leading-edge trigger on the PMT signal. The baseline is calculated event by event as the average signal value over $16 \mathrm{~ns}$ just before the integration window. An adjustable trigger hold-off prevents from retriggering and is set to the same length as the integration window. A second integration window ( $\left.Q_{\text {short }}\right)$ is opened together with the first one to integrate over the fast scintillation component of the signal within $25 \mathrm{~ns}$ ( $42 \mathrm{~ns}$ for the double cell setups). The PSD parameter is then calculated as the fraction of the charge corresponding to the slow component over the total one, i.e. $\operatorname{PSD}=\left(Q_{\text {tot }}-Q_{\text {short }}\right) /\left(Q_{\text {tot }}\right)$. The charge integration windows have been optimized for the two setups independently. For the double cell setup improvements had been made on the precision of the alignment of the light guiding system and PMTs with a higher quantum efficiency have been used. The thus improved scintillation light collection allowed for a longer $Q_{\text {tot }}$ window without integrating dominantly signal noise. The $Q_{\text {short }}$ window has been adjusted accordingly, in order to achieve a maximum 
separation in the PSD values of fission and $\beta$ decay events. Given the slowing down range of the fission fragments in the scintillator (around 20-30 $\mu \mathrm{m}$, calculated with [26]), when the fission occurs in the bulk of the scintillator, the total kinetic energy of both fragments - about $170 \mathrm{MeV}$ in case of ${ }^{235} \mathrm{U}[27]$ - is deposited within the active volume. However, due to strong scintillation quenching for particles with high ionizing power [28], the scintillation light yield of fission fragments is about a factor of 80 smaller than for electrons [18] so that the actually produced light signal is equivalent to that of an electron of only a few $\mathrm{MeV}$ energy. Electrons of a few $\mathrm{MeV}$ on the other hand, originating from $\beta$ decays or from $\gamma$-ray interactions, have a slowing down range of the order of millimeters, thus depositing only a fraction of their energy in the scintillator volume. As a result, a good separation in energy between fission events and electron $/ \gamma$-ray induced signals is achieved. Fig. 4 shows the distribution of PSD and total charge $Q_{\text {tot }}$ resulting from $24 \mathrm{~h}$ of data taking with the ${ }^{235} \mathrm{U}$ single cell target. The electron and fission events as well as certain classes of pile-up can clearly be distinguished (see details in the caption of Fig. 4). As it is evident from the $Q_{\text {tot }}$ projection, a simple energy threshold already provides an efficient separation between $\beta$ decay and fission events. For the ${ }^{235} \mathrm{U}$ targets the alpha activity amounts to about $50 \mathrm{~Bq}$ and is thus negligible. For the ${ }^{233} \mathrm{U}$ target and potential future experiments the alpha activity can reach tens of $\mathrm{kHz}$ and has to be taken into account. Alpha particles also experience strong scintillation quenching and their signal overlaps in energy with the electrons' signal, see Fig. 5, thus a good fission identification is still possible. However, in the choice of the target mass, i.e. of the maximum fission rate for a given neutron flux, the pile-up with $\beta$ - and $\alpha$-decay events has to be taken into account for the fission tagging to be effective. Although the precise pile-up rate depends on the exact length of the signal integration windows (which depends on the scintillation time constants and the light collection efficiency) the overall count rate is limited to about $100 \mathrm{kHz}$ (fission $+\beta$ decay + alpha activity) in order to keep the total pile-up fraction below 1-2\%, for a single target cell. This is the main performance limitation for this type of active target and limits the achievable fission rate to $10-15 \mathrm{kHz}$. For this reason, the double cell setup was developed, to double the fission rate without significantly increasing pile-up.

The event classes indicated in Fig. 4 can be identified using a simplified model based on characteristic pulse shapes of the different ionizing particles. As indicated in the figure, single fission and electron ( $\beta$-decay or $\gamma$-ray induced) events appear in regions I and II, respectively, with (charge|PSD) values around (1400|0.22) and (150|0.11). If two events of identical particles pile-up with only a minor time delay, their energies add up while the PSD remains the same. If the second event occurs in the tail of the first event, which is more likely since this is longer, the PSD is increased to an expected value of

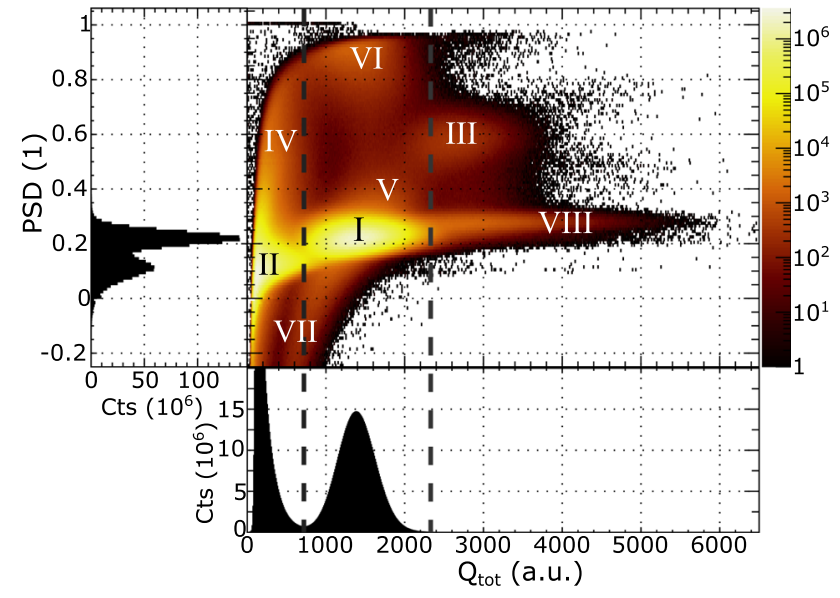

Fig. 4 Distribution of PSD and total charge resulting from $24 \mathrm{~h}$ of ${ }^{235} \mathrm{U}$ fission data in the single cell configuration. The axis projections are shown in the side panels. Different classes of events are labeled in the plot: (I) fission events, (II) electron events, (III) fission-fission pileup, (IV) electron-electron pile-up, (V) fission-electron pile-up, (VI) electron-fission pile-up, (VII) incomplete pulse integration for fission and electron events, respectively, (VIII) tail caused by $\alpha$ particles from ternary fission. The bold dashed lines indicate the $Q_{\text {tot }}$ range which has been used as fission gate for $\gamma$-ray spectroscopy

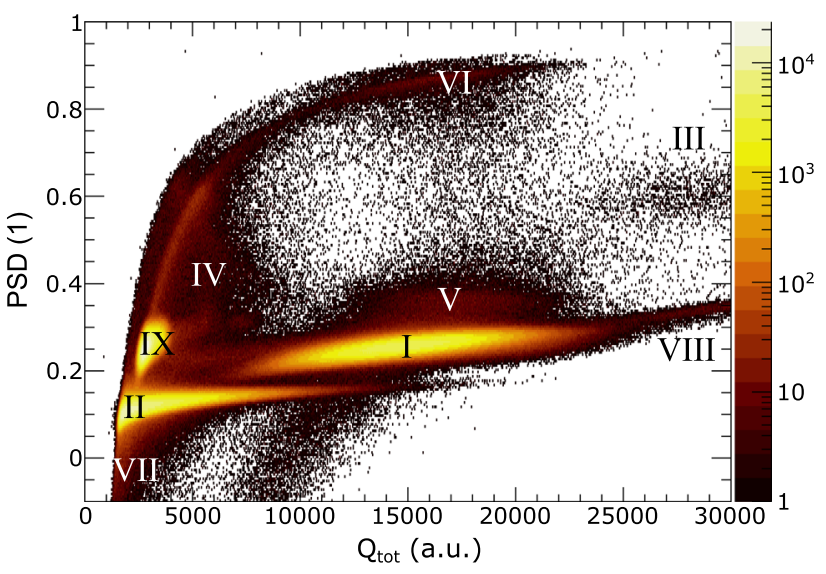

Fig. 5 Distribution of PSD and total charge obtained from the ${ }^{233} \mathrm{U}$ data. The signals corresponding to $\alpha$ decay (region IX) are visible at (charge|PSD) values around (3000|0.25) and can be clearly distinguished from fission and electron events. Other labels correspond to Fig. 4, except that regions IV, V and VI now include pile-up combinations of all three event types: $\alpha$ decay, $\beta$ decay and fission

$(1+\mathrm{PSD}) / 2$. Those appear in region III for fission-fission and in region IV for electron-electron pile-up. If the 2nd event is only partially contained in the integration window, events between the single event and full pile-up distributions occur. Fission-electron pile-up leads only to small changes in energy and PSD of the fission events. They are in region $\mathrm{V}$ with a partial overlap with region I, thus constituting a non-reducible background. For electron-fission pile-up on the other hand the energy is dominated by fission and consequently, if the fission event appears mainly in the tail, can 
lead to high PSD values and the corresponding events appear in region VI. Events in region VII suffer from an incorrect signal reconstruction. If an event occurs just before the trigger hold-off of the preceding event the new trigger appears in the tail of the 2nd signal and the baseline is calculated over the beginning of the actual signal, causing an incorrect baseline subtraction. The tail in region VIII is presumed to have multiple origins. At lower energies close pile-up of two fission events as well as coincident interactions of the fast fission neutrons in the scintillator contribute. The dominant contribution, however, especially at the high energy end, is caused by ternary alpha particle emission during fission. The alpha particles are emitted with energies up to $30 \mathrm{MeV}$ [29] which, considering their lower scintillation quenching compared to fission fragments, leads to high $Q_{\text {tot }}$ values. In $\gamma$-ray coincidences, gated on region VIII, indeed $(\alpha+x \mathrm{n})$ fission fragment pairs dominate over $(x \mathrm{n})$ fragment pairs. The spurious events at higher energies and PSD values indicate three-event pile-up. Given the moderate separation in PSD the event type discrimination (beta vs fission) is only based on an energy interval, for simplicity of data treatment. The lower boundary for a classification as fission is set at the local minimum between the electron- and the fission-distribution. An upper boundary has been set at $+4 \sigma$ from the mean of the approximately Gaussian fission signature to exclude pile-up at higher $Q_{\text {tot }}$ values and the tail region. The corresponding distribution of PSD and total charge resulting from the analysis of the data collected with the ${ }^{233} \mathrm{U}$ loaded target is shown in Fig. 5. The different performances compared to Fig. 4 are due to the above-mentioned improvement on the scintillation light collection. Given the higher specific alpha activity of ${ }^{233} \mathrm{U}$, alpha events are also clearly visible. However, with PSD values comparable to those of fission events but $Q_{\text {tot }}$ values comparable to electron events the fission identification efficiency is not compromised. The general event classes described above (I-VIII) can be well distinguished. The effect of the fission event selection as indicated in Fig. 4 is shown in Fig. 6, where the overall $\gamma$-ray energy spectra with (green) and without (blue) tag condition are compared. The energy spectrum obtained with only the empty target cell in the neutron beam is also shown (red) for comparison. The neutron beam induced background, measured with the empty cell, is dominated by $\gamma$ rays following ${ }^{27} \mathrm{Al}(\mathrm{n}, \gamma)^{28} \mathrm{Al}$ reactions, mainly occurring in the cell windows (sapphire- $\mathrm{Al}_{2} \mathrm{O}_{3}$ ) induced by the direct beam and the cell frame (aluminum) by scattered neutrons. In comparison to a cell filled with loaded scintillator, the most noticeable difference is a peak at $1779 \mathrm{keV}$ (with its Compton continuum) which mainly originates from the $\beta$ decay of the produced ${ }^{28} \mathrm{Al}\left(t_{1 / 2}=2.2 \mathrm{~min}\right.$ [30]). The differences in the intensity of this line in the shown spectra are a result of the ${ }^{28} \mathrm{Al}$ build-up over different irradiation times. As demonstrated in this comparison, by gating on the active target's fission signal the background is reduced

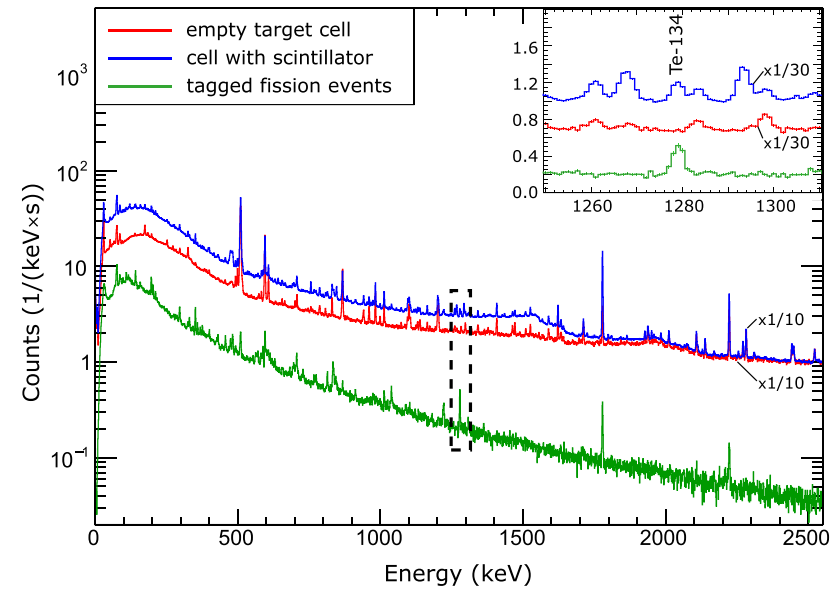

Fig. 6 Comparison of FIPPS $\gamma$-ray energy spectra following the neutron-induced fission of ${ }^{235} \mathrm{U}$. The spectra corresponding to the neutron beam impinging on the empty cell (red) and the cell filled with U-loaded scintillator (blue) are scaled for illustration purposes. The background suppression in the gamma energy spectrum is evident from the spectrum tagged by the PMT signal as fission events (green). The $\gamma$-ray peak corresponding to the $2^{+} \rightarrow 0^{+}$transition in ${ }^{134} \mathrm{Te}$ is highlighted with a dashed box, a zoom on this energy range is shown in the inlay. The different intensities of the peak at $1779 \mathrm{keV}$ originate from different irradiation times and the corresponding build-up of ${ }^{28} \mathrm{Al}$ in the cell materials

considerably and the gain in the peak-over-background ratio for the $\gamma$-ray peaks characteristic of fission events is substantial (see figure caption for more details). Another example of the fission tagging performance on the identification of fission $\gamma$ rays in a multiple $\gamma$-ray coincidence analysis is shown in the gated energy spectra in Fig. 7, explained in the following section.

\subsection{Fission identification efficiency}

The fissile actinide is directly dissolved in the liquid scintillator, providing a $4 \pi$ solid angle coverage for particle detection. Eventual detection efficiency losses can occur for two main reasons: a reduced scintillation light production (e.g. due to fission fragments depositing part of their energy in the cell wall), or an incomplete light collection. In both cases the measured charge $Q_{\text {tot }}$ could fall below the energy threshold selected for the fission gate. The latter effect is mainly governed by the geometry and was taken into account in the present design by keeping the light guiding system as short as possible and with a diameter sufficiently larger than that of the scintillator layer. Event losses due to wall effects are small, given the target geometry, typical stopping ranges of $20-30 \mu \mathrm{m}$ for heavy and light fission fragments, respectively (calculated with [26]), and the fact that the two fragments are emitted back-to-back after thermal neutron induced fission. From simulations in Ref. [18] for different target geometries the expected, relative losses are in the order of $10^{-6}$. Those 


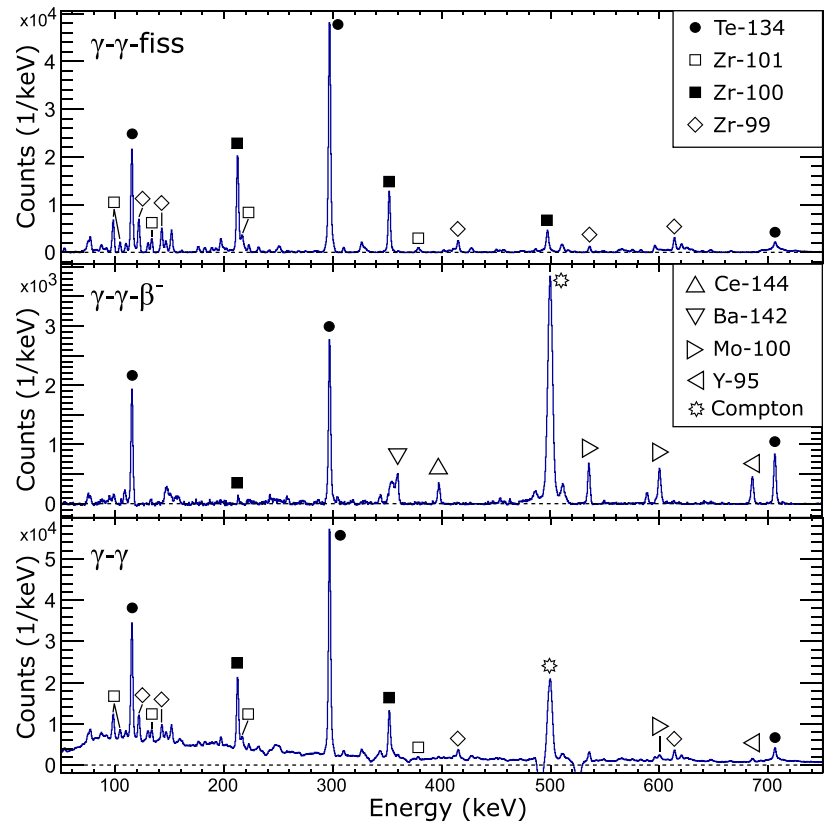

Fig. 7 Comparison of $\gamma$-ray spectra in coincidence with the $1279 \mathrm{keV}$ line of ${ }^{134} \mathrm{Te}$ with and without an additional coincidence condition on the fission or $\beta$ - signal of the active target, respectively. The fission-gated spectrum (top) is dominated by $\gamma$ rays from ${ }^{134} \mathrm{Te}$ and its fission partners corresponding to events with 1,2 and 3 emitted neutrons, namely ${ }^{101} \mathrm{Zr}$, ${ }^{100} \mathrm{Zr}$ and ${ }^{99} \mathrm{Zr}$. The most dominant peaks in the $\beta$ - gated spectrum (center) originate from ${ }^{134} \mathrm{Te}$ and from $\beta$-decay products which also have a transition around $1279 \mathrm{keV}$. The most intense line, at $500 \mathrm{keV}$, is an artefact, caused by Compton-scattering of a $1779 \mathrm{keV} \gamma$ ray from the $\beta$ decay of ${ }^{28} \mathrm{Al}$. It should be mentioned that even though the $\beta$-gated spectrum appears with less intensity, many $\beta$ decay events are below the detection limit of the PMT and are thus not taken into account in this graph. The coincidence spectrum without additional condition (bottom) is the superposition of all fission and $\beta$ decay events and additional random coincidences, leading to a significant Compton background especially at low energies. The spectra correspond to $125 \mathrm{~h}$ of data taking

considerations have been validated by the high fission tagging efficiency obtained in the analysis described below. The experimental fission tagging efficiency of the active target has been determined from the analysis of germanium detector data. This detection efficiency is considered the same for all fission product pairs, given that for each event the summed kinetic energy of both fragments is detected. A particular fission fragment pair can be identified by requiring a coincident detection of two characteristic $\gamma$ rays in the germanium detector array, each belonging to one of the two fission partners. This condition is selective on pure fission events. The total number of detected $\gamma$-ray coincidences of the selected fission partners, $N_{\gamma-\gamma}=\epsilon_{\gamma-\gamma} \cdot N_{\text {pair }}$, depends only on the germanium detector array's detection efficiency at the considered energies $\left(\epsilon_{\gamma-\gamma}\right)$ and the total number of the considered fission fragment pairs produced ( $\left.N_{\text {pair }}\right)$, but it is independent of the active target's performance. The same evaluation can be done when asking for the presence of the fission tag. The fis- sion tagging efficiency $\left(\epsilon_{\text {fiss }}\right)$ can be thus evaluated from the ratio of triple coincidences $N_{\gamma-\gamma-\text { fiss }}=\epsilon_{\text {fiss }} \cdot \epsilon_{\gamma-\gamma} \cdot N_{\text {pair }}$ and double coincidences:

$\epsilon_{\text {fiss }}=\frac{N_{\gamma-\gamma-\text { fiss }}}{N_{\gamma-\gamma}}$.

This ratio is independent of the detection efficiency of the HPGe array and of the target mass. It has been evaluated for the ${ }^{134} \mathrm{Te}-{ }^{100} \mathrm{Zr}$ and ${ }^{90} \mathrm{Kr}-{ }^{144} \mathrm{Ba}$ fission events. As an example, the $\gamma$-ray energy spectra in coincidence with the main gamma transition are shown for the ${ }^{134} \mathrm{Te}-{ }^{100} \mathrm{Zr}$ pair in Fig. 7. From the analysis of the ${ }^{235} \mathrm{U}$ single cell data the fission tagging efficiency, averaged over the two considered fragment pairs, has been obtained as $\epsilon_{\text {fiss }}=97.8 \% \pm 2.5 \%$, assuming symmetric error bars. The uncertainty is not limited by the coincidence counting statistics, but rather by the subtraction of the background (Compton events and random coincidences). The stated efficiency, even though mathematically correct, exceeds the physical limit $(0 \% \leq \epsilon \leq 100 \%)$ already in the $1 \sigma$ interval, but is used for further reference. To harmonize the result with physical boundaries the procedure described in Ref. [31] can be applied. This results in a $68 \%$ confidence interval of $95.3 \%<\epsilon_{\text {fiss }}<99.5 \%$. In the same way the identification efficiencies for both campaigns using the double cell setups $\left(\epsilon_{\mathrm{fiss}}, \mathrm{DC}\right)$ were determined, resulting in $\epsilon_{\text {fiss,DC,U-235 }}=(96.7 \pm 3.3) \%$ and $\epsilon_{\text {fiss,DC,U-233 }}=(98.1 \pm 5.2) \%$, respectively. Both values are found to be in good agreement with the efficiency of the single cell setup. As can be seen in Fig. 4 the present event selection by energy threshold implies that some electron events are mis-identified as fission events. Those have two origins: (i) the highest energetic electron signals extend beyond the energy threshold set for fission identification and (ii) pile-up of fission-electron and electron-fission events overlap with the fission signature region, where the latter may be suppressed by an additional condition on the PSD-value of the signals. If only the energy cutoff is used the fraction of mis-identified electron events in the fission gate can be estimated. For high energetic electron signals, the slope of the electron spectrum can be fitted with an exponential function in the energy range below the fission cutoff value, which then can be extrapolated up to the fission peak. The validity of this approach was tested with data taken immediately after switching off the neutron beam, using the residual $\beta$-decay activity. In this case the extrapolated exponential function agrees well with the beta spectrum. This estimate amounts to a fraction of about $0.4 \%$ relative to the total integral over the fission peak. The pile-up contribution can be estimated assuming a maximum fission rate of $12 \mathrm{kHz}$, six $\beta$ decays per fission and a PMT event time window of 60 ns. Here a simple pile-up estimate following Poisson statistics yields about $0.4 \%$ events, relative to the number of fission events. 


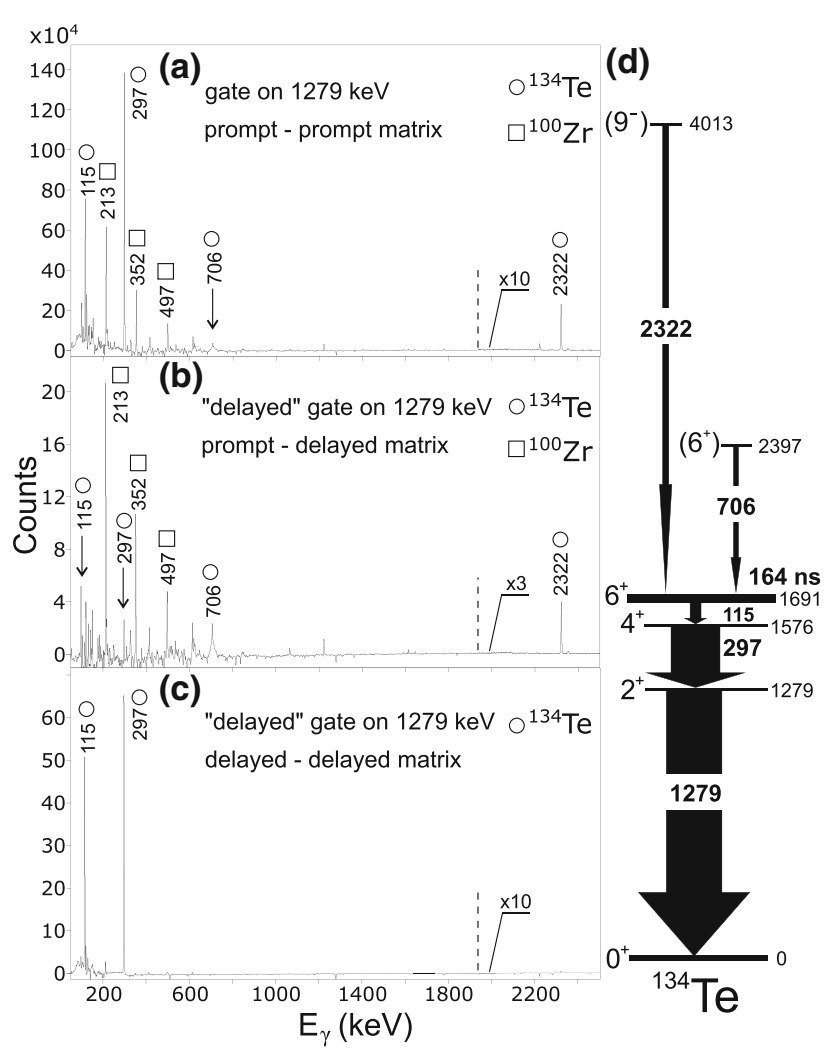

Fig. 8 a-c Gamma-ray spectra in coincidence with the $1279 \mathrm{keV}$ line of ${ }^{134} \mathrm{Te}$ : a Prompt coincidences $(0-150 \mathrm{~ns})$. b Prompt-delayed (150$2000 \mathrm{~ns}$ ) coincidences: the 115 and $297 \mathrm{keV}$ lines located below the isomer are significantly suppressed while the 706 and $2322 \mathrm{keV}$ lines above the isomer are, relatively, enhanced. The same applies to the transitions of the fission partners. Given the relatively short half-life small contributions from delayed transitions are also present in the spectrum. c Delayed-delayed coincidences: only the transitions located below the isomer are present while the lines preceding the isomer or from the fission partners are not. d Partial level scheme of ${ }^{134} \mathrm{Te}$ [34]

Both effects combined lead to an estimated electron event mis-classification of $0.8 \%$, relative to the number of fissions.

\subsection{Impact on the study of isomeric states}

The information provided by the active target, with its good time resolution, can also be exploited to improve the quality of the analysis spectra used in prompt-delayed coincidence studies. This technique (see e.g. Ref. [32] for details) is used at HPGe-detector arrays for the investigation of isomeric states with half-lives in the tens of ns to $\mu$ s range. In this kind of investigations, the $\gamma$-ray energy spectra obtained with different time conditions are analyzed, considering as time reference the "trigger" of the events. In the case of experiments with pulsed beams the trigger is defined by the pulse structure. In contrast, for fission studies at continuous beams or spontaneous sources and in the absence of a fission tag, this condition relies on the multiplicity of triggered detec- tors in a defined prompt time window. In the case of data recorded in list-mode, as it is the case of the present study, this condition can be considered in a versatile way. This was also the case in past neutron-induced fission experiments at ILL, which were part of the EXILL campaign [6]. In order to apply the prompt-delayed gamma coincidence technique for the EXILL configuration, a fission event was identified by the occurrence of at least four $\gamma$ rays within a $200 \mathrm{~ns}$ prompt time window [33] with at least one delayed coincidence (trigger condition denoted P4D1). As an alternative condition was considered a multiplicity of three within $200 \mathrm{~ns}$, followed by at least other three $\gamma$ rays within the following $1500 \mathrm{~ns}$ (P3D3). Both conditions resulted from a study of numerous nuclei with different isomeric half-lives and ensure that the contribution of random coincidences is small. These restrictions on detector multiplicity in the assignment of the events resulted in a significant reduction of the statistics available for the analysis. In contrast, by using an active target the multiple coincidence condition can be replaced by one single information. The strength of the prompt-delayed coincidence analysis of isomeric states is demonstrated on the case of ${ }^{134} \mathrm{Te}$, for prompt-delayed coincidences across the $164 \mathrm{~ns}$ half-life isomeric state (see partial level scheme in Fig. 8d). The spectra in coincidence with the $1279 \mathrm{keV}, 2^{+} \rightarrow 0^{+}$ transition, lying below the isomer, are shown in Fig. $8 \mathrm{a}-\mathrm{c}$ for different coincidence time conditions, taking the active target as a reference. By using a "prompt" time gate $(\mathrm{P})$, in the interval $0-150 \mathrm{~ns}$, the transitions dominating the $\gamma$-ray spectrum are those of ${ }^{134} \mathrm{Te}$ itself and its fission partners ${ }^{99,100,101} \mathrm{Zr}$-as illustrated in Fig. 8a (see also Fig. 7). The transitions above the isomer become dominant in the $\gamma$-ray spectrum when selecting coincidences of a prompt signal with the delayed component (D) of the $1279 \mathrm{keV}$ line, $150-2000 \mathrm{~ns}$ after fission, as shown in Fig. 8b (see figure caption for details). The coincidence time windows were chosen as a compromise between the half-life of the isomer and the contributions from random coincidences. This method provides strong selection criteria, given the selection in energy and time, which can even be improved by applying triple coincidences (i.e. PPD, PDD, DDD). Furthermore, it allows to complete level schemes above and below isomers and, from the time correlations between prompt and delayed $\gamma$-rays, to determine the half-life of the isomeric state. One essential requirement, however, is a precise time information about the fission event. In order to evaluate the impact of the active target on this kind of studies the two triggering schemes were directly compared by sorting the same fission data set, acquired with the active target, twice: once considering the tag information for the establishment of the time coincidences and once using only $\gamma$-ray multiplicity conditions. In both cases PD matrices have been analyzed by selecting the $1279 \mathrm{keV}$ line from ${ }^{134} \mathrm{Te}$ as delayed coincidence signal. The $2322 \mathrm{keV}$ transition located above the isomer in ${ }^{134} \mathrm{Te}$ and the $213 \mathrm{keV}$ line belonging to 
Table 2 Comparison of fission triggering schemes with and without use of the active target. The numbers refer to the prompt coincidences with the $1279 \mathrm{keV}$ transition, depopulating the $164 \mathrm{~ns}$ half-life isomer in ${ }^{134} \mathrm{Te}$. Columns 3 and 4 correspond to the two multiplicity conditions described in the text. Both conditions are considered as minimum conditions, where higher multiplicities are also accepted. Therefore, if evaluated individually, they are not fully distinct and events with at least four prompt and three delayed $\gamma$ rays (P4D3) fulfill both conditions. This overlap (column 5) is subtracted to obtain the total sum in column 6

\begin{tabular}{|c|c|c|c|c|c|c|}
\hline \multirow[t]{2}{*}{ Transition energies (keV) } & \multirow[t]{2}{*}{ With active target } & \multicolumn{4}{|c|}{ Without active target } & \multirow[t]{2}{*}{ Ratio (\%) } \\
\hline & & P4D1 & P3D3 & P4D3 & Total & \\
\hline 2322 & $6706(82)$ & $916(30)$ & $238(15)$ & $100(10)$ & $1054(35)$ & $15.7(6)$ \\
\hline 213 & $69229(263)$ & $6453(80)$ & $1425(38)$ & $517(23)$ & $7361(92)$ & $10.6(1)$ \\
\hline
\end{tabular}

the fission partner ${ }^{100} \mathrm{Zr}$ have been considered for the evaluation of the number of prompt-delayed coincidences. The results of this comparison are listed in Table 2. As reported in the last column, showing the ratio of coincident events in the two cases, the number of prompt-delayed coincidences using the active target is larger by a factor $7-10$ with respect to a trigger condition relying only on the multiple $\gamma$-ray coincidence information.

\section{Summary}

A liquid scintillator based active fission target has been developed for the FIPPS instrument at ILL, which enabled for the first time an efficient suppression of $\gamma$ rays from $\beta$-decaying fission products in high-resolution and high-efficiency $\gamma$-ray spectroscopy of fission fragments at a neutron beam. The use of only thin layers of selected materials and an indirect scintillation light readout guarantee a minimal interference with the neutron beam and thus a minimal $\gamma$-ray background. Campaigns have been performed with thermal neutron induced fission of ${ }^{235} \mathrm{U}$ and ${ }^{233} \mathrm{U}$. Other actinides may in principle be used, with possible limitations due to their radiotoxicity, which renders the handling more difficult, and intrinsic alpha activity, which adds to the overall count rate and pile-up of the active target. In the performed campaigns a good fissionto- $\beta$-decay separation is achieved with a fission identification efficiency of $97.8(25) \%$ and an estimated relative contribution of $\beta$ decays in the fission gate of $0.8 \%$. By using the active target signal as event trigger in the offline data analysis, clean $\gamma$-ray spectra of fission products can be obtained allowing for more accurate spectroscopic studies of neutron-rich nuclei. The gain up to a factor of 10 in statistics in prompt-delayed coincidences analysis for the study of isomeric states has been demonstrated.

Acknowledgements This work benefited from the Horizon2020 program of the European Commission through the FILL2030 project, Grant agreement $\mathrm{N}^{\mathrm{O}}$ 731096. We acknowledge the support of C. Mokry, J. Runke and N. Trautmann at JGU Mainz in the chemical separation of the ${ }^{233} \mathrm{U}$. The authors are grateful for the the technical and administrative support of the ILL's radiation protection service.
Data Availability Statement This manuscript has associated data in a data repository. [Authors' comment: It is accessible according to the ILL's data policy. Information on the modes of access can be found in refs. $[24,25]$ or by contacting the corresponding authors.]

Open Access This article is licensed under a Creative Commons Attribution 4.0 International License, which permits use, sharing, adaptation, distribution and reproduction in any medium or format, as long as you give appropriate credit to the original author(s) and the source, provide a link to the Creative Commons licence, and indicate if changes were made. The images or other third party material in this article are included in the article's Creative Commons licence, unless indicated otherwise in a credit line to the material. If material is not included in the article's Creative Commons licence and your intended use is not permitted by statutory regulation or exceeds the permitted use, you will need to obtain permission directly from the copyright holder. To view a copy of this licence, visit http://creativecomm ons.org/licenses/by/4.0/.

\section{References}

1. J. Eberth, J. Simpson, Prog. Part. Nucl. Phys. 60, 283 (2008). https://doi.org/10.1016/j.ppnp.2007.09.001

2. M.A.C. Hotchkis et al., Phys. Rev. Lett. 64, 3123 (1990). https:// doi.org/10.1103/PhysRevLett.64.3123

3. J.H. Hamilton et al., Prog. Part. Nucl. Phys. 35, 635 (1995). https:// doi.org/10.1016/0146-6410(95)00048-N

4. W. Urban et al., Z. Phys. A 358, 145 (1997). https://doi.org/10. 1007/s002180050291

5. I.Y. Lee, Prog. Part. Nucl. Phys. 38, 65 (1997). https://doi.org/10. 1016/S0146-6410(97)00009-4

6. M. Jentschel et al., J. Instrum. 12, P11003 (2017). https://doi.org/ 10.1088/1748-0221/12/11/p11003

7. R. Krücken et al., Eur. Phys. J. A 10, 151 (2001)

8. T. Kutsarova et al., Phys. Rev. C 58, 1966 (1998). https://doi.org/ 10.1103/PhysRevC.58.1966

9. M. Calviani et al., Nucl. Instr. Methods A 594, 220 (2008). https:// doi.org/10.1016/j.nima.2008.06.006

10. A. Tsinganis et al., Phys. Proc. 64, 130 (2015). https://doi.org/10. 1016/j.phpro.2015.04.017

11. C. Guerrero et al., Eur. Phys. J. A 48, 29 (2012). https://doi.org/ 10.1140/epja/i2012-12029-2

12. C.Y. Wu et al., Nucl. Instr. Methods A 694, 78 (2012). https://doi. org/10.1016/j.nima.2012.07.056

13. G. Rusev et al., Nucl. Instr. Methods A 804, 207 (2015). https:// doi.org/10.1016/j.nima.2015.09.078

14. P.F. Schuster et al., Phys. Rev. C 100, 014605 (2019). https://doi. org/10.1103/PhysRevC.100.014605 
15. J. Scherzinger et al., Appl. Radiat. Isot. 128, 270 (2017). https:// doi.org/10.1016/j.apradiso.2017.05.022

16. C. Michelagnoli et al., EPJ Web Conf. 193, 04009 (2018). https:// doi.org/10.1051/epjconf/201819304009

17. IFIN-HH institute website. http://www.nipne.ro/index.php. Accessed 06 Apr 2020

18. G. Belier et al., Nucl. Instr. Methods A 664, 341 (2012). https:// doi.org/10.1016/j.nima.2011.09.065

19. G. Belier et al., Phys. Rev. C 98, 034612 (2018). https://doi.org/ 10.1103/PhysRevC.98.034612

20. P. Obložinský, B. Pritychenko (eds), Nucl. Data Sheets 148, 1 (2018)

21. N. Dacheux, J. Aupiais, Anal. Chem. 69(13), 2275 (1997). https:// doi.org/10.1021/ac961209r

22. Product Bulletin DF2000 Revision C, January $2017 . \quad \mathrm{https}: / /$ multimedia.3m.com/mws/media/982449O/ $3 \mathrm{mtm}$-specular-film-df2000ma-technical-data-sheet.pdf. Accessed 06 Apr 2020

23. M. Janecek, W.W. Moses, IEEE Trans. Nucl. Sci. 55, 2432 (2008). https://doi.org/10.1109/TNS.2008.2001408

24. https://doi.org/10.5291/ILL-DATA.3-17-24

25. https://doi.org/10.5291/ILL-DATA.3-17-37
26. O.B. Tarasov, D. Bazin, Nucl. Instr. Methods B 266, 4657 (2008). https://doi.org/10.1016/j.nimb.2008.05.110

27. H.W. Schmitt, J.H. Neiler, F.J. Walter, Phys. Rev. 141, 1146 (1966). https://doi.org/10.1103/PhysRev.141.1146

28. J.B. Birks, Proc. Phys. Soc. A 64, 874 (1951). https://doi.org/10. 1088/0370-1298/64/10/303

29. C. Wagemans et al., Phys. Rev. C 33, 943 (1986). https://doi.org/ 10.1103/PhysRevC.33.943

30. M.Shamsuzzoha Basunia, Nucl. Data Sheets 114, 1189 (2013). https://doi.org/10.1016/j.nds.2013.10.001

31. G.J. Feldman, R.D. Cousins, Phys. Rev. D 57, 3873 (1998). https:// doi.org/10.1103/PhysRevD.57.3873

32. Ł.W. Iskra et al., Phys. Rev. C 93, 014303 (2016). https://doi.org/ 10.1103/PhysRevC.93.014303

33. Ł.W. Iskra et al., Europhys. Lett. 117, 12001 (2017). https://doi. org/10.1209/0295-5075/117/12001

34. A.A. Sonzogni, Nucl. Data Sheets 103, 1 (2004). https://doi.org/ 10.1016/j.nds.2004.11.001 